\title{
Conductive, self-healable and reusable
}

\section{poly(3,4-ethylenedioxythiophene)-based}

\section{hydrogels for highly sensitive pressure}

\section{arrays}

Ismael Babeli, ${ }^{\mathrm{a}, \diamond}$ Guillem Ruano, ${ }^{\mathrm{a}, \diamond}$ Jordi Casanovas, ${ }^{\mathrm{b}}$ Maria-Pau Ginebra, ${ }^{c, d, e}$ Jose García-Torres ${ }^{c, d, *}$ and Carlos Alemán ${ }^{\mathrm{a}, \mathrm{d}, \mathrm{e}, \text {, }}$

a Departament d'Enginyeria Química, EEBE, Universitat Politècnica de Catalunya, C/ Eduard Maristany, 10-14, 08019, Barcelona, Spain

${ }^{\mathrm{b}}$ Departament de Química, Escola Politècnica Superior, Universitat de Lleida, c/Jaume II no. 69, Lleida E-25001, Spain

${ }^{\mathrm{c}}$ Biomaterials, Biomechanics and Tissue Engineering Group, Department of Materials Science and Metallurgical Engineering, Universitat Politècnica de Catalunya (UPC), 08930 Barcelona, Spain

${ }^{\mathrm{d}}$ Barcelona Research Center in Multiscale Science and Engineering, Universitat Politècnica de Catalunya, 08930 Barcelona, Spain

${ }^{\mathrm{e}}$ Institute for Bioengineering of Catalonia (IBEC), Barcelona Institute of Science and Technology (BIST), Baldiri Reixac 10-12, 08028 Barcelona Spain

${ }^{\diamond}$ These authors contributed equally to this work *jose.manuel.garcia-torres@ upc.edu and carlos.aleman@upc.edu 


\begin{abstract}
Although challenging, the preparation of pure conducting polymer $(\mathrm{CP})$ hydrogels as conductive flexible networks for developing high-performance functional platforms is an outstanding alternative to conventional approaches, as for example those based on the cross-linking of insulating polymer with $\mathrm{CP}$ segments and the simple utilization of CPs as fillers of insulating hydrogel networks. In this work, we propose the employment of poly(3,4-ethylenedioxythiophene) : poly(styrenesulfonate) (PEDOT:PSS) to prepare conductive hydrogels by partially replacing the PSS dopant by alginate (Alg) chains, which is energetically favoured. The capacity of Alg chains to be electrostatically crosslinked by $\mathrm{Ca}^{2+}$ ions has allowed us to obtain hydrogels with good electrical percolation response and mechanical properties. Hydrogels were prepared in a very simple one-step process by adding $\mathrm{CaCl}_{2}$ to different mixtures of PEDOT:PSS and alginic acid (1:3, $1: 1$ and 3:1). After structural, chemical and physical characterization, the 1:3 PEDOT/Alg hydrogel was moulded to fabricate stretchable touch-pressure sensor arrays, which exhibited fast response and good spatial resolution of the pressure distribution. In addition, PEDOT/Alg hydrogel is self-healable which allowed us to prepare reusable pressure sensors (i.e. devices that can be reprocessed to be used in its original application) thanks to the reversibility of the noncovalent $\mathrm{Ca}^{2+}$ crosslinks. Reusable devices are in opposition to reclaimed and recycled devices as they are no longer use for the same application because the materials lose their properties. With our hydrogel we are a step closer to a circular economy by allowing the reuse of electronic devices and reducing electronic waste worldwide. Moreover, the superior performance of PEDOT/Alg hydrogel opens up its utilization as efficient and flexible pressure sensors for wearable human-electronic interfaces, in which reusability would be an added value.
\end{abstract}




\section{Introduction}

Conducting polymer $(\mathrm{CP})$ hydrogels are usually prepared by combining an insulating polymer, which forms three-dimensional networks that have the capacity to absorb large amount of water, and a $\mathrm{CP}$ that mainly provides electrical conductivity and/or electrochemical activity. ${ }^{1-5}$ However, the properties of the resulting hydrogels are typically compromised when CPs are added as simple fillers in hydrogel networks, this inherent limitation sometimes restricting their applicability. A much better approach, but also more complex from a chemical point of view, is to combine the unique properties of the insulating polymer and the $\mathrm{CP}$ by a proper coupling reaction between segments of such materials to produce an integrated network of cross-links. ${ }^{6-11}$ The most advantageous approach, which is very recent, consists on the formation of pure CP hydrogels to produce outstanding conducting networks that can be integrated in highperformance functional platforms. ${ }^{12-14}$

Poly(3,4-ethylenedioxythiophene) (PEDOT) is one of the most studied CPs because of its excellent properties (i.e. great environmental stability, electrical conductivity, electrochemical activity, thermoelectric behaviour and high specific capacitance). ${ }^{15-18}$ In particular, poly(styrenesulfonate) (PSS)-doped PEDOT is a very attractive material with good film forming properties, moderate to high conductivity, high visible light transmittance, excellent stability and, therefore, used in a wide number of technological applications (e.g. sensors, electrodes for supercapacitors, soft actuators, and as hole injection/extraction material in organic optoelectronics). ${ }^{19-23}$ In PEDOT:PSS, the CP domains are embedded in an electronically insulating PSS matrix loosely cross-linked by hydrogen bonding. ${ }^{23}$

Due to its interesting properties, efforts on the preparation of pure CP hydrogels have been mainly focused on PEDOT:PSS..$^{13,14,24}$ In a very recent study, Zhao and 
coworkers $^{13}$ engineered a simple process to produce pure CP hydrogels by interconnecting networks of PEDOT:PSS nanofibrils. This was achieved by mixing dimethyl sulfoxide (DMSO) into PEDOT:PSS aqueous dispersions followed by controlled dry-annealing and rehydration. The resulting hydrogels were proved to fulfil the properties desired for bioelectronic applications. Besides, Shi and coworkers ${ }^{14}$ prepared CP hydrogels by thermal treatment of commercial PEDOT:PSS suspensions in diluted $\mathrm{H}_{2} \mathrm{SO}_{4}$ followed by partial removal of the PSS with concentrated $\mathrm{H}_{2} \mathrm{SO}_{4}$ and purification by washing with deionized water. The resulting hydrogels were dried and used to prepare highly conducting fibres, which were subsequently employed to fabricate flexible supercapacitors. In both cases, the transformation of PEDOT:PSS into a conducting hydrogel required several steps including both chemical and physical treatments. $^{13,14}$ More recently, Bao and co-workers developed an electrochemical gelation method for patterning PEDOT:PSS hydrogels on conductive templates using a sacrificial metal layer. ${ }^{24} \mathrm{~A}$ few months ago, Khademhosseini and coworkers ${ }^{25}$ reported the spontaneous formation of PEDOT:PSS hydrogel by injecting a mixture of the CP suspension with 4-dodecylbenzenesulfonic acid, which is a cytotoxic surfactant. The resulting hydrogel fibers were found to be self-healing.

In very recent studies PEDOT:PSS has been combined with other polymers to create stretchable and conducting systems. Wang et al. ${ }^{26}$ constructed a 3D segregated structure in the carboxylated styrene butadiene latex films using PEDOT:PSS, which led to highly stretchable composite with sensitivity towards touchless stimuli. Also, an all inkjet-printed sensor for hydrazine detection has been prepared using a PEDOT:PSS electrode functionalized with $\mathrm{ZnO}$ and encapsulated in a Nafion matrix. ${ }^{27}$ Wang et al. ${ }^{28}$ blended PEDOT:PSS with graphene oxide on flexible polyethylene terephthalate for applications based on the piezoresistive properties of the resulting composite. Zhou et 
al. $^{29}$ nanostructured fibres anchoring a thin layer of polyaniline on PEDOT:PSS hydrogel for fibre supercapacitors. Teo et $a l .{ }^{30}$ prepared PEDOT:PSS / ionic liquid hydrogels by microreactive inkjet printing for bioelectronic applications. The main disadvantage of these hybrid hydrogels refers to the cytotoxicity of the component that is mixed with PEDOT:PSS (e.g. polyaniline and ionic liquids are very cytotoxic materials), which hinders their utilization for some biomedical applications. Moreover, many of the mentioned hydrogels were not self-healing.

Herein, we report a new PEDOT hydrogel that combines high conductivity, flexibility and self-healing property with the outstanding capacity of reutilization, which in the context of this work should be understood as the capacity to be recycled for the same application (i.e. without loss of properties) by re-constituting it after complete disintegration. This hydrogel is rapidly formed in a very simple one step process from commercial PEDOT:PSS through the replacement of PSS dopant polyanions by alginate (Alg) chains. More specifically, the simply addition of a $\mathrm{CaCl}_{2}$ solution to a mixture of PEDOT:PSS and alginic acid (AA) directly results in the formation of a hydrogel, hereafter named PEDOT/Alg-h. Furthermore, this hydrogel can be undone by disassembling the polymer chains through the replacement of $\mathrm{Ca}^{2+}$ by monovalent ions like $\mathrm{Na}^{+}$and, subsequently, reconstituted by adding $\mathrm{CaCl}_{2}$ again.

Alg is a biocompatible and biodegradable copolymer with homopolymeric blocks of 1,4-linked $\beta$-D-mannuronate and its C-5 epimer, $\alpha$-L-guluronate, covalently linked in different sequences. The mannuronate / guluronate ratio and length of each block depend on the source of the Alg. ${ }^{31}$ Alg is frequently employed in the form of hydrogel in biomedicine, mainly in wound healing, in vitro cell culture, drug delivery and tissue engineering. $^{32}$ 
Taking advantage of the properties of the PEDOT hydrogel obtained by replacing PSS by Alg in a $\mathrm{Ca}^{2+}$-containing medium, a reusable pressure sensor array with high capability for detecting both location and pressure of touches, as well as with excellent response time, has been prepared. Results demonstrate that the novel hydrogel, named PEDOT/Alg, can be considered as an ideal material for many applications (e.g. textile devices, including wearable ones, keyboards, touchscreens and human-machine interfaces) in which the reconstitution of the sensor, instead of its replacement, could be considered as the most appropriated alternative.

\section{Results and discussion}

\section{Conceptualization}

The energetic associated to the substitution of PSS polyanions by Alg chains in PEDOT:PSS has been evaluated using Density Functional Theory (DFT) calculations at the M06L/6-31G(d,p) level. The aqueous medium was described using the Polarizable Continuum Model (PCM), which is a well-stablished Self-Consistent Reaction Field method. Calculations were conducted on simple model systems involving a PEDOT chain with 4 or 8 repeat units and a charge of +0.5 per repeat unit (i.e. 4 -EDOT ${ }^{+2}$ or 8 EDOT $^{+4}$ ) complexed to: $i$ ) a PSS chain with 2 or 4 repeat units and a charge of -1 per repeat unit (i.e. 2-SS ${ }^{2-}$ or $4-\mathrm{SS}^{4-}$ ); or ii) an $\mathrm{Alg}$ chain with two (one mannuronate and one guluronate residues; $m=n=1$ in Scheme 1) or four (two mannuronate and two guluronate residues; $m=n=2$ in Scheme 1) units, blocked at the ends with methyl groups, and a charge of -1 per unit (i.e. $2-\mathrm{Alg}^{2-}$ or $4-\mathrm{Alg}^{4-}$ ). It is worth noting that the half positive charge used for each PEDOT repeat unit, which has been taken from previous measures of the doping level, ${ }^{33}$ reflects the characteristic delocalized electronic distribution of heterocyclic CPs, while the negative charge of each PSS or Alg unit is 
specifically localized on the corresponding sulfonate or carboxylate group, respectively. In order to search for the most favourable interaction between the model molecules involved in each calculated complex, different relative orientations were considered as starting points for geometry optimizations (i.e. at least 5 different starting points were considered for each complex). Results are summarized in Figure 1.

The binding energies associated to the formation of PEDOT:PSS complexes indicate that this assembly process is more favoured in vacuum than in aqueous solution (Figure 1a). Water shields both the delocalized charges of PEDOT and the localized charges of the PSS, reducing the strength of the electrostatic interactions between the two species. Besides, the strength of the interaction increases with the size of the model molecules. Thus, the binding energy becomes 2.6 (vacuum) and 1.4 (aqueous solution) times more attractive when the size of the model molecules used to represent PEDOT and PSS increases from $4-\mathrm{EDOT}^{+2}$ and $2-\mathrm{SS}^{2-}$ to $8-\mathrm{EDOT}^{+4}$ and $4-\mathrm{SS}^{4-}$. As shown in Figure $1 \mathrm{~b}$, which displays the most stable $8-\mathrm{EDOT}^{+4}: 4-\mathrm{SS}^{4-}$ complex, the sulfonate groups preferentially interact with the oxidized thiophene rings.

Although the binding energies calculated for PEDOT:Alg (Figure 1c) show trends similar to those described for PEDOT:PSS, the influence of the size of the model is significantly more pronounced. More specifically, the binding energy becomes 2.9 and 2.5 times more attractive in vacuum and aqueous solution, respectively, when the size of model oligomers doubles. Consequently, the binding energies are more attractive for $8-\mathrm{EDOT}^{+4}: 4-\mathrm{Alg}^{4-}$ than for $8-\mathrm{EDOT}^{+4}: 4-\mathrm{SS}^{4-}(-93.2$ and $-19.0 \mathrm{kcal} / \mathrm{mol}$ in vacuum and aqueous solution, respectively), confirming that the substitution of PSS by Alg in PEDOT:PSS complexes is favoured from a thermodynamic point of view. This has been attributed to the flexibility of Alg chains, which permits the re-orientation of the carboxylate groups to achieve stronger electrostatic interactions with the thiophene ring. 
Figure 1d, which depicts the most stable $8-\mathrm{EDOT}^{+4}: 4-\mathrm{Alg}^{4-}$ complex, shows that the carboxylate rings of $4-\mathrm{Alg}^{4-}$ directly confront the oxidized thiophene rings. This structural re-orientation is more difficult in PEDOT:PSS since the bulk phenyl groups easily induce severe steric repulsions.

\section{Preparation of PEDOT/Alg hydrogels}

As DFT calculations indicated that PEDOT $\cdots$ Alg interactions are energetically favoured with respect to PEDOT $\cdots$ PSS, substitution of PSS by Alg was directly attempted using commercial PEDOT:PSS. Three different PEDOT:PSS/Alg hydrogels, named 1:3, 1:1 and 3:1, were prepared, this choice allowing to roughly scan the properties of the whole range of compositions. For this purpose, equal volumes of a 1.3 wt. \% PEDOT:PSS aqueous dispersion and a $1.3 \mathrm{wt} \%$ or $3.9 \mathrm{wt} \%$ AA water solution were mixed at room temperature with vigorous stirring for $20 \mathrm{~min}$. Hereafter, the mixtures coming from $1.3 \mathrm{wt} \%$ and $3.9 \mathrm{wt} \%$ AA solutions are denoted 1:1 PEDOT:PSS/AA and 1:3 PEDOT:PSS/AA. Also, the 3:1 PEDOT:PSS/AA mixture was prepared using $1.3 \mathrm{wt} \% \mathrm{AA}$ and increasing three times the volume of $1.3 \mathrm{wt} \%$ PEDOT:PSS with respect to that of AA. These mixtures were transformed in hydrogels by immersing silicon rubber moulds of well-defined dimensions, which were previously filled with the corresponding PEDOT:PSS/AA mixture, into a $\mathrm{CaCl}_{2} 3$ wt.\% aqueous solution for $10 \mathrm{~min}$; or by depositing the corresponding mixture onto a substrate (e.g. cover glass) and, subsequently, adding $\mathrm{CaCl}_{2} 3$ wt.\% aqueous solution. The excess of AA and the leaving PSS chains (i.e. those replaced by Alg) were eliminated by thoroughly washing the formed hydrogel with abundant water. Hydrogels prepared using this simple process, which is sketched in Scheme 1, are denoted 3:1, 1:1 or 1:3 PEDOT/Alg-h. 
In the dispersion, the PEDOT:PSS complex is usually described as a micro-gel system, where a PEDOT-rich center is surrounded by excessive PSS. In addition, PSS plays the role of a primary dopant agent for PEDOT. This is because of the electronic structure of negatively charged sulfonate groups that is stabilized by the aromatic phenyl rings. Substitution of PSS by Alg, which is also a very soluble polyelectrolyte, is expected to maintain the dispersion of the $\mathrm{CP}$ in the solution. This explains the observation of PEDOT-rich domains surrounded by Alg-rich in the prepared hydrogels (see next-subsection). However, the role of Alg as primary dopant agent is expected to be worse than that of PSS since in the former the negative charges are not stabilized by aromatic moieties. This is also consistent with the moderate conductivity of PEDOT/Alg-h, as shown below.

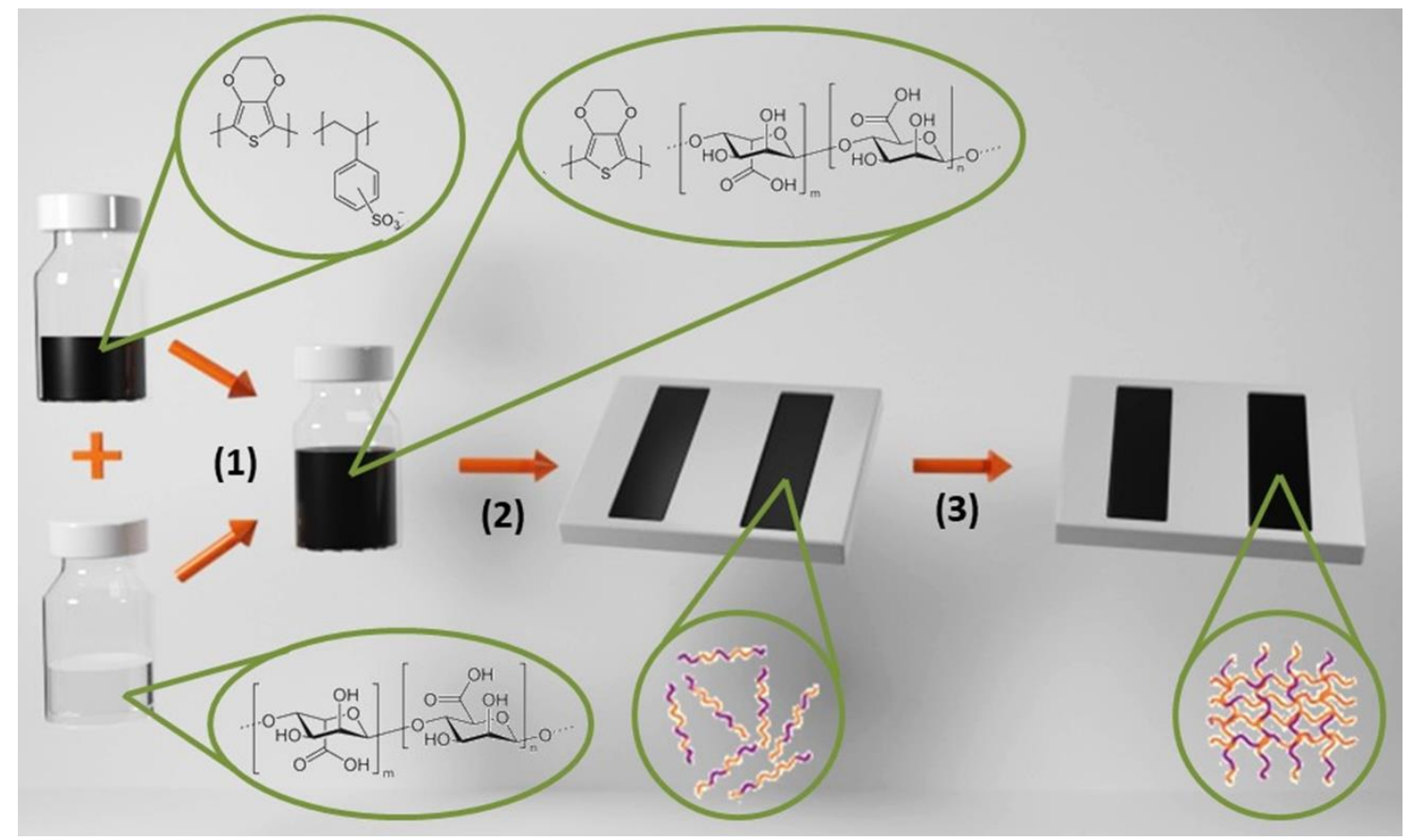

Scheme 1. Procedure used to prepare PEDOT/Alg-h. (1) Mixing of alginate solution and PEDOT:PSS dispersion and stirring for $20 \mathrm{~min}$. (2) Pouring the mixture in the silicone rubber mould. (3) Cross-linking in $3 \mathrm{wt} . \% \mathrm{CaCl}_{2}$ solution for $3 \mathrm{~min}$. 
Besides, hydrogels derived from pure PEDOT:PSS and pure AA, hereafter named PEDOT-h and Alg-h, respectively, were used as controls. More specifically, PEDOT-h was obtained applying the $\mathrm{H}_{2} \mathrm{SO}_{4}$ treatment described by Shi and coworkers (see experimental section for details), ${ }^{14}$ while Alg-h was derived from a 1.3 wt $\%$ AA solution using the procedure described for PEDOT/Alg-h.

\section{Characterization of PEDOT/AIg hydrogels}

Figure 2a compares the FTIR spectra of PEDOT-h, Alg-h and 3:1, 1:1 and 1:3 PEDOT/Alg-h. In addition of the broad band at $\sim 3400 \mathrm{~cm}^{-1}$ (not shown), which is attributed to the $\mathrm{O}-\mathrm{H}$ stretching, the spectrum of Alg-h displays absorption bands assigned to carbonyl $(\mathrm{C}=\mathrm{O})$ asymmetric and symmetric stretching at 1598 and $1418 \mathrm{~cm}^{-}$ ${ }^{1}$, respectively, and the $\mathrm{C}-\mathrm{O}-\mathrm{C}$ stretching at $1028 \mathrm{~cm}^{-1} \cdot{ }^{34}$ On the other hand, PEDOT-h shows the characteristic absorption bands of the $\mathrm{CP}$ at $1564 \mathrm{~cm}^{-1}$ for the $\mathrm{C}=\mathrm{C}$ stretching in the thiophene ring, 1270 and $1122 \mathrm{~cm}^{-1}$ for the vibrations of the fused dioxane ring, and $862 \mathrm{~cm}^{-1}$ for the stretch of the $\mathrm{C}-\mathrm{S}$ bond in the thiophene ring. Moreover, the bands at 1162 and $1060 \mathrm{~cm}^{-1}$ have been attributed to the $\mathrm{S}-\mathrm{O}$ and $\mathrm{S}-$ phenyl vibrations of residual PSS chains (i.e. those that were not removed by the acid treatment ${ }^{14}$ ). The FTIR spectra recorded for 3:1, 1:1 and 1:3 PEDOT/Alg-h are consistent with the concentration of PEDOT and AA in the starting mixtures. Most importantly, in all cases the bands associated to PSS are residual, reflecting that the substitution of this component by Alg chains is almost complete. Raman spectra recorded for the different compositions of PEDOT/Alg-h show that PEDOT bands predominate over Alg bands due to the resonance Raman effect (Figure 2b), which increases the intensity of the bands of the material when the laser energy coincides with the frequency of the 
electronic transition of the sample. ${ }^{35,36}$ Detailed discussion of the bands is provided in the Supplementary Information.

To get some insights into the microstructure of the materials, we conducted scanning electron microscopy (SEM) studies of lyophilized hydrogels. Alg-h exhibits a homogeneous interconnected macroporous "open cell" structure with irregular large pores of $43 \pm 7 \mu \mathrm{m}$ in diameter (Figure 2c). Similarly, PEDOT-h forms a 3D network, even though in this case the pore diameter shows great variability (i.e. from 1 to $46 \mu \mathrm{m}$ ) with an average value of $12 \pm 9 \mu \mathrm{m}$ (Figure 2d). On the other hand, Alg-h is easy handling and shows mechanical integrity and stiffness (Figure S1a). These properties are due to the stability provided by the binding between the $\mathrm{Ca}^{2+}$ ions and the carboxylate groups of the guluronate blocks of two adjacent polymer chains (i.e. "eggbox" model). Instead, the mechanical behaviour of PEDOT-h is very poor, breaking by its own weight (Figure S1b) and making it difficult to handle even with the help of tweezers. This feature has been attributed to the weakness of the cross-linking interactions and explains that a drying process, which enhanced the strength of such interactions, was required to obtain mechanically strong porous PEDOT-h fibres. ${ }^{14}$

SEM micrographs of 3:1, 1:1 and 1:3 PEDOT/Alg-h are displayed in Figures 2e-f. The three systems present similar macroporous open structures, but the pore size is significantly affected by both the PEDOT content and the degree of replacement of PSS by $\operatorname{Alg}$ (i.e. $20 \pm 5,9 \pm 2$ and $68 \pm 11 \mu \mathrm{m}$ for the 3:1, 1:1 and 1:3 hydrogels, respectively). Additional SEM micrographs and energy dispersive X-ray (EDX) spectra, which indicate that the atomic compositions of the hydrogels are consistent with those of the PEDOT:PSS/AA feeding mixtures, are displayed in Figure S2. As occurred for PEDOT-h, the mechanical integrity and the easy of handling of 3:1 PEDOT:Alg-h are very poor (Figure S1c), which has been attributed to a combination of two factors. First, 
the high concentration of $\mathrm{CP}$ weakens cross-linking interactions, as happens in PEDOT$\mathrm{h}$, which are responsible for the consistency of the material. Second, PEDOT and Alg chains are segregated in different domains, as is suggested by phase imaging atomic force microscopy (AFM). Recorded images show very significant phase shift signal, distinguishing between bright (PEDOT domains) and dark (Alg domains) separated patterns (Figure 3a).

Instead, 1:1 and 1:3 PEDOT/Alg-h show easy handle-ability and processability (Figures 2h-i), which justify the preparation of hydrogels with higher Alg content. Besides, height and phase-contrast AFM images show that the surface topography (i.e. the root mean square roughness, RMS $\mathrm{R}_{\mathrm{q}}$ ) and the microstructure (i.e. the distribution of PEDOT and Alg chains in the hydrogel) of PEDOT/Alg-h changes with the composition (Figure 3). While in the 1:1 hydrogel the $\mathrm{Alg}$ and $\mathrm{CP}$ chains are homogeneously distributed, as is shown by phase AFM images (Figure 3b), Alg chains form abundant domains of micrometric size in the 1:3 hydrogel (Figure 3c). However, in both cases, the content of Alg chains is high enough to form strong ionic cross-links in which the $\mathrm{Ca}^{2+}$ cations connect the carboxylate groups of different but close $\mathrm{Alg}$ chains. Indeed, the mechanical integrity, texture and, in general, the aspect of 1:1 and 1:3 PEDOT/Alg-h (Figures $2 \mathrm{~h}-\mathrm{i}$ ) is similar to that of Alg-h (Figure S1a). The only exception to this resemblance is the color, which is dominated by the dark blue color of PEDOT. On the other, the RMS $\mathrm{R}_{\mathrm{q}}$ increases with the Alg content, suggesting that the latter defines the surface topography.

The water uptake capacity (Eq S1) of 1:1 and 1:3 PEDOT/Alg-h is $6392 \% \pm 115 \%$ and $3640 \% \pm 147 \%$, indicating that the swelling of these hydrogels decreases with the content of Alg. This has been attributed to strong ionic $\mathrm{Ca}^{2+}$ mediated crosslinks between neighbouring alginate chains, which restrict the molecular movement in the 1:3 
hydrogel. Instead, $\mathrm{Ca}^{2+}$-alginate crosslinks are less abundant in the 1:1 PEDOT:Alg-h, facilitating alginate chains relaxation and water diffusion. Obviously, the dimensional stability of hydrogels increases with decreasing swelling ratio and, therefore, the stability of 1:3 PEDOT/Alg is expected to be superior.

Based on the aforementioned mechanical integrity, easy of handling and dimensional stability, the 1:3 hydrogel was selected for the evaluation of the electrical and electrochemical properties and, subsequently, the fabrication of a reusable pressure sensor array.

\section{Electrical and electrochemical properties of 1:3 PEDOT/Alg-h}

Electrical and electrochemical properties were measured using hydrogel strips of $5 \times$ $3 \times 0.3 \mathrm{~cm}^{3}$ that were prepared in silicone rubber moulds, as shown in Figure $4 \mathrm{a}$. After removing the excess of water, the electronic conductivity $(\sigma)$ of 1:3 PEDOT/Alg-h was determined to be $\sigma=7.9( \pm 0.3) \cdot 10^{-5} \mathrm{~S} / \mathrm{cm}$. This value is one order of magnitude higher than that obtained for Alg-h and similar to those of soft composites obtained by chemically polymerizing CPs inside Alg-h networks. ${ }^{37}$ However, it is much lower than those reported for pure PEDOT hydrogels that ranged from 0.46 to $8.8 \mathrm{~S} / \mathrm{cm}$, depending on the characteristics of the treatment with concentrated $\mathrm{H}_{2} \mathrm{SO}_{4} \cdot{ }^{14}$ The conductivities of 1:1 and 3:1 PEDOT/Alg-h, which were determined for sake of completeness, were $\sigma=$ $5.1( \pm 0.5) \cdot 10^{-3}$ and $8.7( \pm 0.6) \cdot 10^{-2} \mathrm{~S} / \mathrm{cm}$, respectively. The progressive reduction of the conductivity with increasing Alg content has been attributed to the detrimental effect of micrometric polysaccharide-rich domains on the conduction paths, as suggested by phase imaging AFM (Figure 3c).

The ionic conductivity of the 1:3 PEDOT/Alg-h was also determined using electrochemical impedance spectroscopy (EIS) as the high-water content can contribute 
to the total hydrogel conductivity. Accordingly, the impedance was measured for frequencies ranging from $10^{-1}$ to $10^{4} \mathrm{~Hz}$ using a previously reported through-plane impedance cell. ${ }^{38}$ Figure S3 displays the collected impedance $(Z)$ data for three independent replicas as Nyquist plots. The hydrogel showed a typical semicircle in the high frequency region, whose diameter represents the charge-transfer resistance of the interface $\left(R_{c t}\right)$. In all cases, the diameter is very small with an average value of $R_{c t}=48.9$ $\pm 9.8 \mathrm{k} \Omega / \mathrm{cm}^{2}$, indicating that 1:3 PEDOT/Alg-h possesses a high ionic conductivity (ca. $\left.10^{-5} \mathrm{~S} / \mathrm{cm}\right)$. Besides, the straight ascending line at low frequencies with an angle higher than $45^{\circ}$ has been attributed to diffusion of ionic species from the hydrogel to the electrode.

As a complement to AFM images, transmission electron microscopy (TEM) was performed not only for negatively stained hydrogels (using 1\% uranyl acetate (UAc) solution) (Figure 4b) but also for unstained hydrogels (Figure 4c). TEM images of the stained samples show dark regions corresponding to the anionic sites of Alg that were selectively stained with UAc. These higher contrast regions correspond to Alg-rich domains, which are clearly distinguished from the bright regions with a high content of PEDOT (grey). Thus, TEM micrographs of the unstained samples (Figure 4c) show that the latter zones are dominated by the remarkable electron scattering properties of PEDOT. In fact, the contrast of PEDOT-rich domains in unstained samples is relatively similar to that of Alg-rich domains negatively stained with UAc. In spite of this segregation, both TEM and AFM images suggest that this separation is not complete in 1:3 PEDOT/Alg-h. Thus, PEDOT-rich domains contain a fraction of Alg and viceversa, which is essential to preserve stable and repetitive conduction paths across the samples (as proved below). 
The electrical percolation response of 1:3 PEDOT/Alg-h was evaluated by monitoring the change in resistivity as a function of the applied pressure. For this purpose, hydrogels were maintained in the rubber moulds shown in Figure 4a and coated with a thin rubber sheet (thickness: $1 \mathrm{~mm}$ ). Individual cylindrical weights with a nominal mass between $1 \mathrm{~g}$ and $100 \mathrm{~g}$ were carefully deposited on the rubber coating, avoiding direct contact with the hydrogel, and the resistivity was measured using a multimeter that was directly connected to the hydrogel. As shown in Figure 5a, the resistivity decreases linearly with increasing loading, evidencing that the hydrogel is able to detect very low pressures (i.e. as low as $6 \cdot 10^{-5} \mathrm{~Pa}$ ). Repeatability was observed when independently prepared hydrogels were employed. This reproducibility is reflected in Figure 5a, which displays the calibration curves of three independent hydrogels, indicating that 1:3 PEDOT/Alg-h exhibits an outstanding electrical percolation response that reminds the called "piezoresistive response" of PEDOT:PSS/latex ${ }^{26}$ and PEDOT:PSS/graphene oxide ${ }^{28}$ composites.

Cyclic voltammograms recorded for the 1:3 PEDOT/Alg-h (Figure 5b) in a three electrode system in the potential window from 0.0 to $0.8 \mathrm{~V}$ at different scan rates do not exhibit a rectangular shape, reflecting a deviation from the quasi-reversible electrochemical double layer capacitive behaviour typically observed for PEDOT films. ${ }^{39,40}$ However, the current density varies linearly with the square root of the scan rate, as is reflected in Figure 5c for the current density at the reversal (anodic current density, $j_{a}$ ) and the initial potential (cathodic current density, $j_{c}$ ). This regime indicates that the electrochemical processes at 1:3 PEDOT/Alg-h are controlled by diffusion.

Figure $5 \mathrm{~d}$ shows galvanostatic charge-discharge curves between 0.0 and $0.8 \mathrm{~V}$ at a current density of $20 \mu \mathrm{A} / \mathrm{cm}^{2}$. Charge and discharge profiles are linear and, therefore, curves exhibit a typical triangular shape. Although the voltage drop at the beginning of 
the discharging step is very small $($ i.e. $<0.05 \mathrm{~V})$, charge and discharge processes are not symmetric. Specifically, the discharge is twice faster than the charge, reducing the coulombic efficiency of the system. Moreover, the low discharge times and therefore the low capacitance limit the utilization of the 1:3 PEDOT/Alg-h for the construction of efficient supercapacitors. Instead, porous fibres obtained by drying PEDOT:PSS hydrogel fibres treated with $\mathrm{H}_{2} \mathrm{SO}_{4}$ showed a very high Coulombic efficiency (i.e. the charge step was faster than the discharge one). In summary, although 1:3 PEDOT/Alg-h exhibits electrochemical activity, its properties are more appropriated for the fabrication of highly sensitive pressure sensors than for the production energy storage devices.

\section{Preparation of PEDOT-based pressure sensor array and proof of concept}

In order to investigate the potential sensing applications of 1:3 PEDOT/Alg-h, four $5 \times 1 \times 0.2 \mathrm{~cm}^{3}$ hydrogel strips were distributed in two crossed layers separated by a 1 mm-thick silicone rubber film acting as dielectric. The schematic design of the $2 \times 2$ pressure sensor array with the dimensions of each component is sketched in Figure 6a, while the silicone rubber mould used to prepare the two hydrogel strips of each layer is displayed in Figure 6b. Hydrogel strips were contacted by copper wires (wire diameter $=0.1 \mathrm{~mm}$ ) for detection of pressure through a control device. The wires were place inside the moulds and before the preparation of the hydrogels to assure good connection after cross-linking. Figure 6c exhibits a photograph (left) of the two separated layers, each one consisting in a rubber mould with two hydrogel strips prepared by filling each mould with $1 \mathrm{~mL}$ of the 1:3 PEDOT:PSS/AA mixture and immersing the whole later into a $\mathrm{CaCl}_{2} 3$ wt.\% aqueous solution. After drying the excess of water from the surface of the formed hydrogels, the pressure sensor was assembled forming a $2 \times 2$ array with two crossed layers separated by a thin silicone rubber film (Figure 6c, right). 
The evaluation of the prepared array as stretchable touch-pressure sensor was first conducted by demonstrations of controlled illumination of light emitting diodes (LEDs) of different colours (i.e. yellow, blue, green and red). Figure 6d shows a schematic diagram of the voltage divider circuit used to transform the input voltage $\left(\mathrm{V}_{\text {in }}\right)$ into the output voltage $\left(\mathrm{V}_{\text {out }}\right)$ by pressing the hydrogel. Thus, $\mathrm{V}_{\text {in }}$ is affected by the change in resistance of the strip hydrogel when touched, varying from $Z_{1}$ to $Z_{2}$. The change in the voltage is controlled via an Arduino ${ }^{\circledR}$ Leonardo platform that is connected to the pressure sensor array and the different LEDs, as is sketched in Figure 6d.

The microcontroller is responsible for detecting changes in $\mathrm{V}_{\text {out }}$ through the pins and turn on the corresponding LED, thus obtaining a tactile pressure sensor with spatial resolution. Figure 6e illustrates the excellent sensing capacity of the tactile pressure sensor array, which exhibits very fast response and good spatial resolution of the pressure distribution through the outstanding electrical percolation response of hydrogel strips. As it is shown, LEDs light up depending on the location of the pressure exerted by touching the device.

\section{Self-healing and reusability}

Hydrogels stabilized by ionic bonds can be self-healable by modulating the formation and disruption of reversible electrostatic interactions between oppositely charged moieties. Electrostatic interactions can occur between oppositely charged polymers or through ionic bridges between charged polymers mediated by oppositely charged ions. ${ }^{41,42}$ An example of the latter are skin inspired conductive self-healable hydrogels with 3D printability, ${ }^{41}$ which were achieved through dynamic ionic interactions between the carboxylic groups of poly(acrylic acid) and ferric ions. Also, 
Alg-h made of negatively charged alginate chains that cross-link into a hydrogel through divalent ions, belongs to this class of self-healing hydrogels. ${ }^{43,44}$

Figure 7a provides optical evidence of the self-healing ability of 1:3 PEDOT/Alg-h. When a hydrogel strip was cut into two halves by a scalpel and the freshly generated surfaces were brought into contact to allow autonomous healing, the segments merged very rapidly into one piece, adhering to each other with well-bonded interface in less than $1 \mathrm{~min}$. After $5 \mathrm{~min}$, the fused hydrogel can be lifted with tweezers or suspended between the two surfaces. On the other hand, the conductivity of the self-healed hydrogel is practically identical to that of the original one, $\sigma=7.9( \pm 0.3) \cdot 10^{-5} \mathrm{~S} / \mathrm{cm}$, demonstrating its excellent stability and reproducibility for practical applications. Successive cut and healing steps were carried out on the hydrogel at different locations, the restoration of the electrical conductivity confirming the repeatable electrical selfhealing (Figure 7b).

Figure 7c shows nominal strain vs stress curves in tensile of 1:3 PEDOT/Alg-h strips that were submitted to successive cut and healing steps at the same location. The Young moduli of the pristine and the self-healed 1:3 PEDOT/Alg-h are in the range of 0.5-3.0 MPa, which are several orders of magnitude lower than those of conventional polymers. Besides, the elastic limit is higher for the self-healed hydrogels than for the pristine one. This has been attributed to the dynamics of the $\mathrm{Ca}^{2+}$-mediated crosslinks, which favours the formation of entanglements between Alg-rich domains at the damaged regions. On the other hand, the strain $(e)$ of the hydrogel is maintained at around $30 \%$ until the $3^{\text {rd }}$ $4^{\text {th }}$ cut-healing steps, depending on the sample. After this, the breakage is systematically observed at $e \approx 15 \%$, indicating that the self-healing capacity of the same interfaces is limited. This should be attributed to the effects of the cut-healing steps on the distribution of charged groups at the healed interfaces. Thus, coulombic repulsions at 
such interfaces probably increase with the number of cut-healing steps due to the accumulation of $\mathrm{Ca}^{2+}$ and Alg chains. This favours the loss of stretchability and the rupture.

On the hand, the reusability of functional devices based on conductive hydrogels is still an unresolved challenge. It would be highly desirable that hydrogel-based electronic devices, such as pressure sensors, that have worn out and fails to work properly for different reasons (e.g. mechanical failure and dehydration) could be reused for the same electronic functionality using a simple procedure. This reusable capacity is significantly important for addressing the problem of recycling and reprocessing electronic devices, especially taking into account the massive electronic waste produced worldwide. In addition, the reutilization of electronic products reduces the number of items to be manufactured, which in turn has a favourable impact on the conservation of natural resources and raw materials necessary for the manufacture of such products. In addition, once marketed, it is expected that reusable electronic products capable of maintaining their functionality will help consumers reduce costs compared to the purchase of single-use electronic items.

Although the development of reusable hydrogels has been recently claimed by different authors, ${ }^{45,46}$ in such studies the term "reusability" was not associated to disassembling and reprocessing steps but to regeneration and recycling. Liu et al. $^{45}$ restored the swelling property of polyacrylamide-alginate hydrogels for wearable force sensors, alleviating the fall of functionality induced by the structure shrinkage associated to the loss of water. However, this reusability, which was based on a simple rehydration by casting in water, should be considered simply as a clean regeneration treatment to preserve the functionality of the hydrogel. Instead, Pereira and coworkers, ${ }^{46}$ proved the recycling ability of cellulose-based hydrogel sticker films, which were 
applied as electrolyte-gated sensors in transistors. In this case, the material was completely dissolved and, subsequently, reconstituted with the resulting solution. However, the electrical performance of the reprocessed hydrogel as electrolyte-gated sensor was worse than that of pristine hydrogel, suggesting that its recycling to a different application would be the most appropriated.

In this work the concept of reusable hydrogels has been clearly established and differentiated from regenerated and recyclable hydrogels. Thus, the conception of reusable hydrogels is not based on treatments to preserve the functionality of already formed hydrogels (i.e. regeneration) or the utilization of reconstituted hydrogels for applications other than the initial one due to a loss of properties (i.e. recycling) but on the use of the reprocessed hydrogel in its original application. Figure $7 \mathrm{~d}$ shows the steps followed in this work to disassemble an already prepared 1:3 PEDOT/Alg-h and, subsequently, reprocess it maintaining its properties. First, the hydrogel is submerged in a PBS solution with slight magnetic stirring until disassembly occurs and its components are incorporated into the solution. After this, the solution is centrifuged at $11000 \mathrm{rpm}$ for $45 \mathrm{~min}$ at $4^{\circ} \mathrm{C}$ to recover both PEDOT and Alg. The mixture is resuspended with $3 \mathrm{~mL}$ of water and, then, stirred to homogenize. The solution is poured into the silicone rubber mould and, finally, the crosslinking is carried out by adding $\mathrm{CaCl}_{2} 3$ wt.\% aqueous solution. The reprocessed hydrogel can be reutilized as pressure sensor without loss of properties (Figure S4).

\section{Conclusions}

In summary, we demonstrated a flexible pressure sensor with fast response and good spatial resolution of the pressure distribution using a PEDOT-based hydrogel. This hydrogel was obtained by mixing a commercial PEDOT:PSS aqueous dispersion with 
an AA aqueous solution. The substitution of PSS by Alg as dopant agent, which is thermodynamically favoured as proved by DFT calculations, promotes the gelation process when the mixture enters in contact with a $\mathrm{CaCl}_{2}$ solution. After characterization of the structure and properties of hydrogels derived from $3: 1,1: 1$ and 1:3 PEDOT:PSS/AA mixtures, the 1:3 hydrogel was found to be the most appropriated for the fabrication of sensors because of its mechanical integrity, ease of handling and dimensional stability. The internal structure of the 1:3 hydrogel with Alg-rich domains contacting conductive PEDOT-containing networks provides electrical percolation response and self-healing behaviour. The spatial resolution was proved using a $2 \times 2$ array as stretchable touch-pressure sensor that was able to control the illumination of LEDs. The sensor was reusable because of the reversibility of noncovalent $\mathrm{Ca}^{2+}$-based crosslinks. Thus, the hydrogel was disassembled and, subsequently, reprocessed to be reused for the same application, which represents a step forward in terms of sustainability overcoming concepts such as regenerability and recyclability. Overall, demonstrations of the performance of 1:3 PEDOT/Alg-h as reusable sensitive pressure sensor further uncover the promising perspective of hydrogel wearable electronics for the cutting-edge human-electronic interfaces.

\section{Conflicts of interest}

There are no conflicts to declare.

\section{Acknowledgements}

Authors acknowledge MINECO-FEDER (RTI2018-098951-B-I00) and Agència de Gestió d'Ajuts Universitaris i de Recerca (2017SGR359) for financial support. Support for the research of M.-P.G. and C.A. was received through the prize "ICREA 
Academia" for excellence in research funded by the Generalitat de Catalunya. J.G.-T. acknowledges the Serra Hunter program of the Generalitat de Catalunya. 


\section{References}

1. F. Chen, Q. Chen, Q. Song, H. Lu and M. M. Ma, Adv. Mater. Interfaces, 2019, 6, 1900133.

2. C. C. Shih, Y. C. Lin, M. Y. Gao, M. Wu, H. C. Hsieh, N. L. Wu and W. C. Chen, J. Power Sourc., 2019, 426, 205-215.

3. D. L. Gan, L. Han, M. H. Wang, W. S. Xing, T. Xu, H. P. Zhang, K. F. Wang, L. M. Fang and X. Lu, ACS Appl. Mater. Interfaces, 2018, 10, 36218-36228.

4. A. R. Spencer, A. Primbetova, A. N. Koppes, R. A. Koppes, H. Fenniri and N. Annabi, ACS Biomat. Sci. Eng., 2018, 4, 1558-1567

5. Z. X. Deng, Y. Guo, X. Zhao, P. X. Ma and B. L. Guo, Chem. Mater., 2018, 30, $1729-1742$.

6. J. Chen, Q. Peng, T. Thundat and H. Zeng, Chem. Mater., 2019, 311, 24553-4563.

7. S. Q. Hu, L. Zhou, L. J. Tu, C. Dai, L. Fan, K. J. Zhang, T. T. Yao, J. Q. Chen, Z. G. Wang, J. Xing, R. M. Fu, P. Yu, G. X. Tan, J. Q. Du and C. Y. Ning, J. Mater. Chem. B, 2019, 7, 2389-2397.

8. C. Y. Yang, P. F. Zhang, A. Nautiyal, S. H. Li, N. Liu, J. L. Yin, K. L. Deng and X. Y. Zhang, ACS Appl. Mater. Interfaces, 2019, 11, 4258-4267.

9. P. Chakraborty, T. Guterman, N. Adadi, M. Yadid, T. Brosh, L. Adler-Abramovich, T. Dvir and Gazit, ACS Nano, 2019, 13, 163-175.

10. B. L. Guo, A. Finne-Wistrand and A. C. Albertsson, Chem. Mater., 2011, 23, 12541262.

11. B. L. Guo, A. Finne-Wistrand, A. C. Albertsson, J. Polym. Sci.: Polym. Chem.. 2011, 49, 2097-2105. 
12. K. Zhou, Y. He, Q. Xu, Q. Zhang, A. Zhou, Z. Lu, L.-K. Yang, Y. Jiang, D. Ge, X. Y. Liu and H. Bai, ACS Nano, 2018, 12, 5888-5894.

13. B. Lu, H. Yuk, S. Lin, N. Jian, K. Qu, J. Xu and X. Zhao, Nat. Commun., 2019, 10, 1043.

14. B. Yao, H. Wang, Q. Zhou, M. Wu, M. Zhang, C. Li and G. Shi, Adv. Mater., 2017, 29, 1700974

15. O. Bubnova, Z. U. Khan, H. Wang, S. Braun, D. R. Evans, M. Fabretto, P. HojatiTalemi, D. Dagnelund, J. B. Arlin, Y. H. Geerts, S. Desbief, D. W. Breiby, J. W. Andreasen, R. Lazzaroni, W. M. Chen, I. Zozoulenko, M. Fahlman, P. J. Murphy, M. Berggren and X. Crispin, Nat. Mater., 2014, 13, 190.

16. L. V. Kayser and D. J. Lipomi, Adv. Mater., 2019, 31, 1806133.

17. L. D. Sappia, E. Piccinini, W. Marmisolle, N. Santilli, E. Maza, S. Moya, F. Battaglini, R. E. Madrid and O. Azzaroni, Adv. Mater. Interf., 2017, 17, 1700502.

18. L. B. Groenendaal, F. Jonas, D. Freitag, H. Pielartzik and J. R. Reynolds, $A d v$. Mater., 2000, 12, 481-494.

19. J. E. Lim, S. M. Lee, S. S. Kim, T. W. Kim, H. W. Koo and H. Kim, Sci. Rep., $2017,7,14685$.

20. M. Y. Teo, N. Kim, S. Kee, B. S. Kim, G. Kim, S. Hong, S. Jung and K. Lee, ACS Appl. Mater. Inferfaces, 2017, 9, 819-826.

21. S. Taccola, F. Greco, E. Sinibaldi, A. Mondini, B. Mazzolai and V. Mattoli, $A d v$. Mater., 2015, 27, 1668-1675.

22. Y. Li, M. Liu, Y. Li, K. Yuan, L. Xu, W. Yu, R. Chen, X. Qiu and H.-L. Yip, Adv. Energy Mater., 2017, 7, 1601499.

23. T. Stöcker, A. Köhler and R. Moos, J. Polym. Sci., Part B: Polym. Phys., 2012, 50, 976-983. 
24. V. R. Feig, H. Tran, M. Lee, K. Liu, Z. Huang and L. Beker, Adv. Mater., 2019, 31, 1902869.

25. S. Zhang, Y. Chen, H. Liu, Z. Wang, H. Ling, C. Wang, J. Ni, B. Çelebi-Saltik, X. Wang, X. Meng, H.-J. Kim, A. Baidya, S. Ahadian, N. Ashammakhi, M. R. Dokmeci, J. Travas-Sejdic and A. Khademhosseini, Adv. Mater., 2020, 32, 1904752.

26. Z. Wang, T. Wang, M. Zhuang and H. Xu, ACS Appl. Mater. Interfaces, 2019, 11, 45301-45309.

27. T. Beduk, E. Bihar, S. G. Surya, A. N. Castillo, S. Inal and K. N Salama, Sens. Actuators B Chem., 2020, 306, 127539.

28. J.-C. Wang, R. Subhra-Karmakar, Y.-J. Lu, S.-H. Chan, M.-C. Wu, K.-J. Wu, K.-J. Lin, C.-K- Chen, K.-C. Chen, K.-C. Wei and Y.-H. Hsu, ACS Appl. Mater. Interfaces, 2019, 11, 34305-34315.

29. . Q. Q. Zhou, W. L. Teng, J. H. Jin, L. Sun, P. Hu, H. Y. Li, L. Z. Wang and J. S. Wang, Electrochim. Acta, 2020, 334, 135530.

30. M. Y. Teo, N. RaviChandran, N. Kim, S. Kee, L. Stuart, K. C. Aw and J. Stringer, ACS Appl. Mater. Interfaces, 2019, 11, 37069-37076.

31. K. Y. Lee and D. J. Mooney, Prog. Polym. Sci., 2012, 37, 106-126.

32. I. Braccini and S. Pérez, Biomacromolecules, 2001, 2, 1089-1096.

33. C. Ocampo, R. Oliver, E. Armelin, C. Alemán and F. Estrany, J. Polym. Res., 2006, 13, 193-200.

34. S. Hua, H. Ma, L. Xun, H. Yang and A. Wang, Int. J. Biol. Macromol., 2010, 46, $517-523$.

35. A. Shakoor and T. Z. Rizvi, J. Raman Spectrosc., 2010, 41, 237-240. 
36. R. V. Salvaterra, L. G. Moura, M. M. Oliveira, M. A. Pimienta and A. J. G. Zarbin, J. Raman Spectrosc., 2012, 43, 1094-1100.

37. N. Bodenberger, D. Kubiczeklrina, I. Abrosimova, A. Scharm, F. Kipper, P. Walther and F. Rosenau, Biotechnol Rep., 2016, 12, 6-12.

38. F. Müller, C. A. Ferreira, D. S. Azambuja, C. Alemán and E. Armelin, J. Phys. Chem. B 2014, 118, 1102-1112.

39. I. Gualandi, D. Tonelli, F. Mariani, E. Scavetta, M. Marzocchi and B. Fabroni, Sci Rep., 2016, 6, 35419.

40. D. Aradilla, D. Azambuja, M. T. Estrany, C. A. Ferreira and C. Alemán, J. Mater. Chem., 2012, 22, 13110-13122.

41. M. A. Darabi, A. Khosrozadeh, R. Mbeleck, Y. Liu, Q. Chang, J. Jiang, J. Cai, Q. Wang, G. Luo and M. Xing, Adv. Mater., 2017, 29, 1700533.

42. C. Y. Shao, M. Wang, L. Meng, H. L. Chang, B. Wang, F. Xu, J. Wang and P. B. Wan, Chem. Mater., 2018, 30, 3110-3121.

43. A. D. Augst, H. J. Kong and D. J. Mooney, Macromol. Biosci., 2006, 6, 623-633.

44. J. V. Alegre-Requena, M. Häring, R. P. Herrera and D. Díaz-Díaz, New J. Chem., 2016, 40, 8493-8501.

45. H. Liu, M. Li, C. Ouyang, T. J. Lu, F. Li and F. Xu, Small, 2018, 14, 1801711.

46. I. Cunha, R. Barras, P. Grey, D. Gaspar, E. Fortunato, R. Martins and L. Pereira, Adv. Funct. Mater., 2017, 27, 1606755. 


\section{CAPTIONS TO FIGURES}

Figure 1. (a) Binding energies $\left(\Delta \mathrm{E}_{\mathrm{b}}\right.$, in $\left.\mathrm{kcal} / \mathrm{mol}\right)$ associated to the formation of 4$\mathrm{EDOT}^{2+}: 2-\mathrm{SS}^{2-}$ (plain and black) and $8-\mathrm{EDOT}^{4+}: 4-\mathrm{SS}^{4-}$ (italic and red) in vacuum (gp) and aqueous solution (aq) as determined by PCM-M06L/6-31G(d,p) calculations. (b) Lowest energy complex obtained for $8-\mathrm{EDOT}^{4+}: 4-\mathrm{SS}^{4-}$. (c) Binding energies $\left(\Delta \mathrm{E}_{\mathrm{b}}\right.$, in $\mathrm{kcal} / \mathrm{mol}$ ) associated to the formation of $4-\mathrm{EDOT}^{2+}: 2-\mathrm{Alg}^{2-}$ (plain and black) and 8 $\mathrm{EDOT}^{4+}: 4-\mathrm{Alg}^{4-}$ (italic and red) in vacuum (gp) and aqueous solution (aq) as determined by PCM-M06L/6-31G(d,p) calculations. (d) Lowest energy complex obtained for 8$\mathrm{EDOT}^{4+}: 4-\mathrm{Alg}^{4-}$.

Figure 2. (a) FTIR and (b) Raman spectra of Alg-h, PEDOT-h and 3:1, 1:1 and 1:3 PEDOT/Alg-h. Characteristic bands of Alg (light blue), PEDOT (black) and PSS (green) are indicated by dashed lines and discussed in the text. SEM micrographs of (c) Alg-h, (d) PEDOT-h, (e) 3:1, (f) 1:1 and (g) 1:3 PEDOT/Alg-h. Photographic images of (h) 1:1 and (i) 1:3 PEDOT/Alg-h as prepared (top) and handled with tweezers (bottom).

Figure 3. Height (left) and phase (right) AFM images of (a) 3:1, (b) 1:1 and (c) 1:3 PEDOT/Alg-h. RMS roughness $\left(\mathrm{R}_{\mathrm{q}}\right)$ values are indicated.

Figure 4. (a) Preparation of 1:3 PEDOT/Alg-h pieces in a silicon rubber mold. (b,c) TEM micrographs of 1:3 PEDOT/Alg-h samples (b) stained with UAc and (c) unstained.

Figure 5. (a) Relative change of the resistivity with the pressure (results for three independent experiments are shown). (b) Cyclic voltammograms recorded from 0.0 to $0.8 \mathrm{~V}$ at different scan rates. (c) Variation of the anodic (black) and cathodic (red) current density ( $j_{\mathrm{a}}$ and $j_{\mathrm{c}}$, respectively) against the square root of the scan rate. (d) 
Galvanostatic charge-discharge curves (fifth cycle) recorded from 0 to $0.8 \mathrm{~V}$ at a current density of $20 \mu \mathrm{A} / \mathrm{cm}^{2}$ (results for three independent samples are shown).

Figure 6. (a) Schematic design of the pressure sensor and dimensions of each component. (b) Silicon rubber mold used to prepare each layer of the pressure sensor. (c) Two silicon rubber layers containing 2 hydrogel strips with embedded copper electrodes before (left) and after assembly into a $2 \times 2$ crossed array (right). (d) Sketches showing the circuit used to calculate the output voltage $\left(\mathrm{V}_{\text {out }}\right)$ via an Arduino® UNO platform $\left(\mathrm{V}_{\text {in }}\right.$ is the input voltage, while $\mathrm{Z}_{1}$ and $\mathrm{Z}_{2}$ are the resistance of non-touched and touched hydrogels) and the connections among the microcontroller, the pressure sensor array and the yellow, blue, green and red LEDs. (e) Demonstration of the sensing capacity of the tactile pressure sensor array in terms of location and pressure of touches.

Figure 7. (a) Demonstration of the autonomous self-healing by the 1:3 PEDOT/Algh. The healed hydrogel can hold its own weight. (b) Electrical conductivity of the hydrogel as prepared and after successive cut-healing steps at different locations. (c) Strain-stress curves of the hydrogel as prepared and after successive cut-healing steps at the same location. (d) Disassembly-reprocessing steps for the reutilization of the 1:3 PEDOT/Alg-h as pressure sensor: dissolution of the hydrogel in PBS; centrifugation to recover both PEDOT and Alg; re-suspension and homogenization in water; and crosslinking in a silicon rubber mold by adding $\mathrm{CaCl}_{2} 3$ wt.\% aqueous solution. 
(a) $\begin{array}{r}\Delta \mathrm{E}_{\mathrm{b}}=-238.2 \\ \Delta \mathrm{E}_{b}=-632.6\end{array}$

$$
\text { PEDOT (gp) + PSS (gp) } \longrightarrow \text { PEDOT : PSS (gp) }
$$$$
\begin{gathered}
\Delta \mathrm{E}_{\mathrm{b}}=-26.3 \\
\Delta \mathrm{E}_{b}=-36.0 \\
\text { PEDOT (aq) + PSS (aq) } \stackrel{\text { PEDOT : PSS (aq) }}{\longrightarrow}
\end{gathered}
$$

(b)

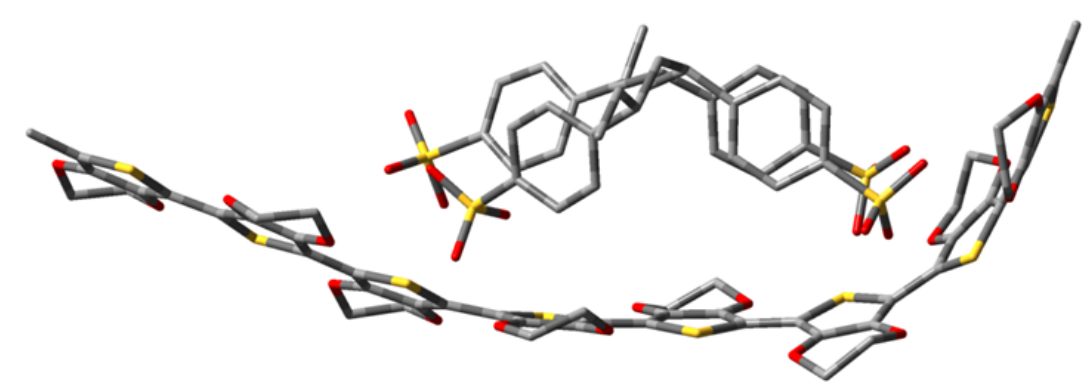

(c) PEDOT (gp) + Alg (gp) $\stackrel{\Delta \mathrm{E}_{b}=-251.0}{\longrightarrow}$ PEDOT : Alg (gp)

$$
\begin{gathered}
\Delta \mathrm{E}_{\mathrm{b}}=-21.7 \\
\Delta \mathrm{E}_{b}=-55.0
\end{gathered} \text { PEDOT (aq) + Alg (aq) } \stackrel{\text { PEDOT : Alg (aq) }}{\longrightarrow}
$$

(d)

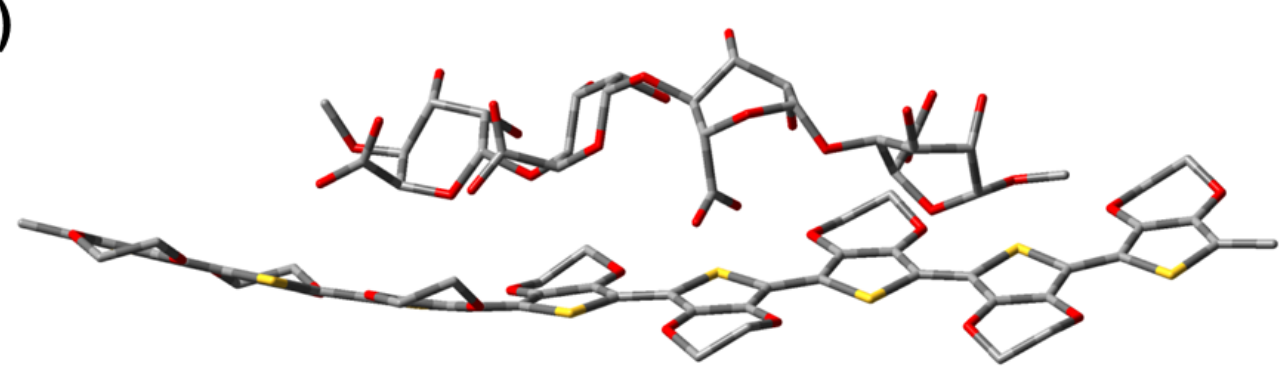

Figure 1 
(a)

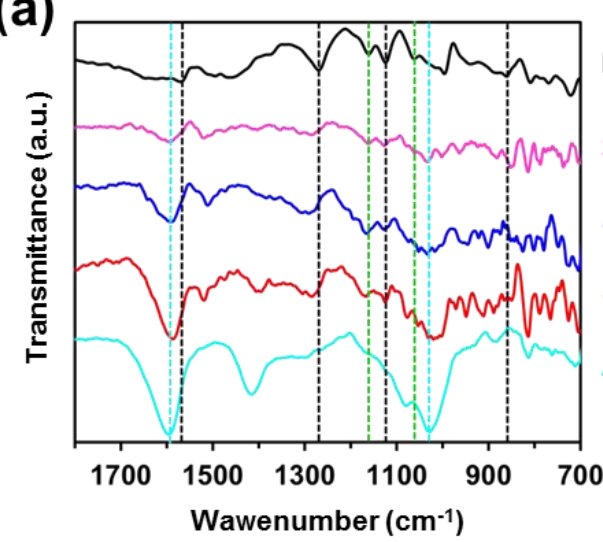

(c)

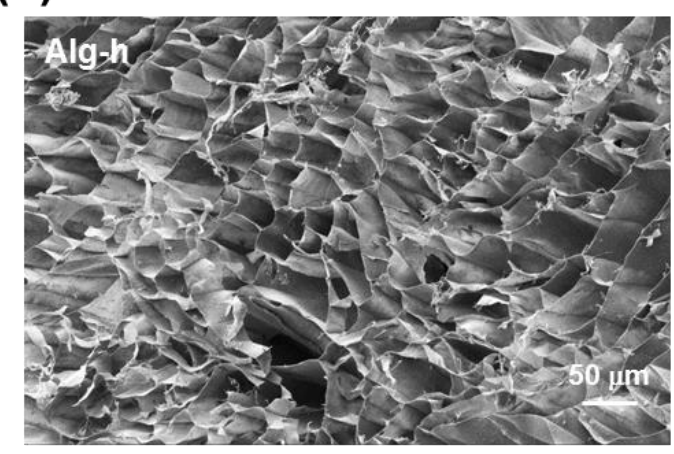

(e)

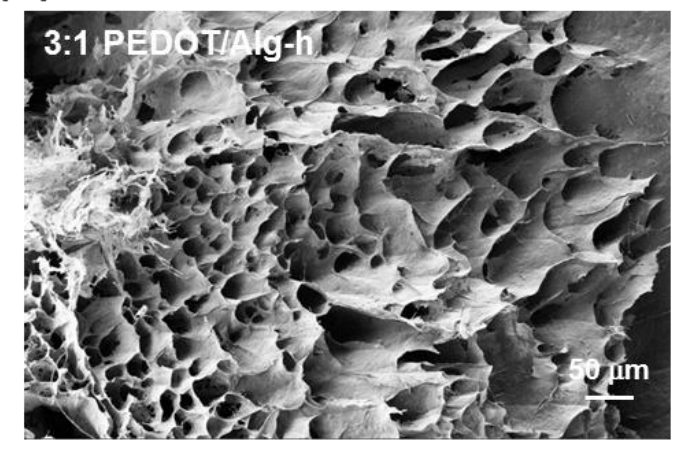

(g)

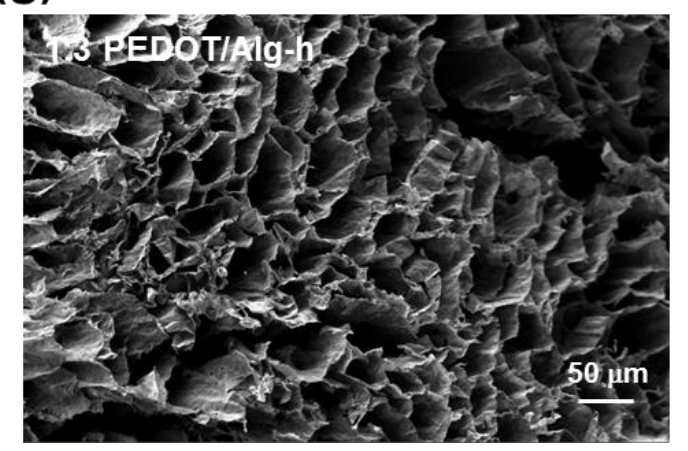
Alg-h (b) PEDOT-h 3:1 PEDOT/Alg-h 1:1 PEDOT/Alg-h 1:3 PEDOT/Alg-h

(d)
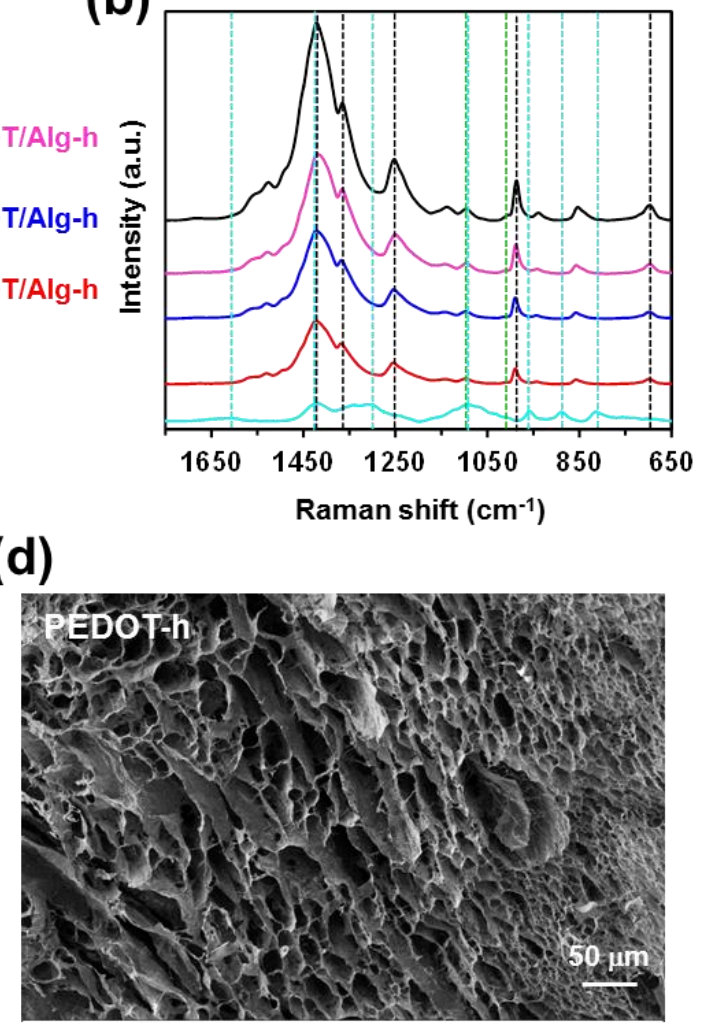

(f)

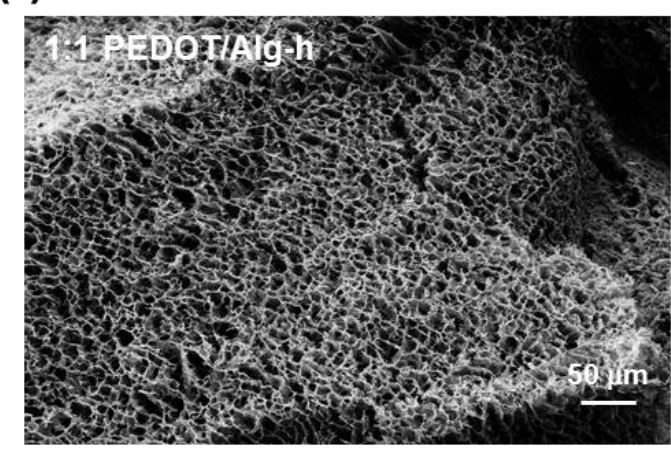

(h)

(i)

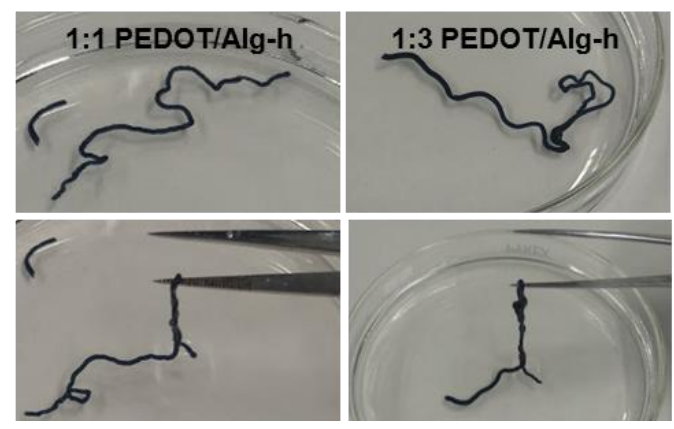

Figure 2 
(a)
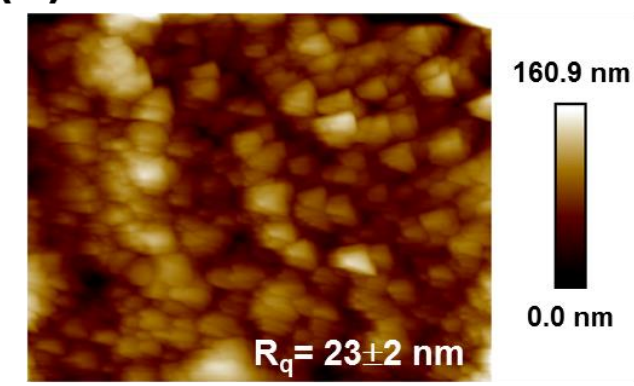

(b)
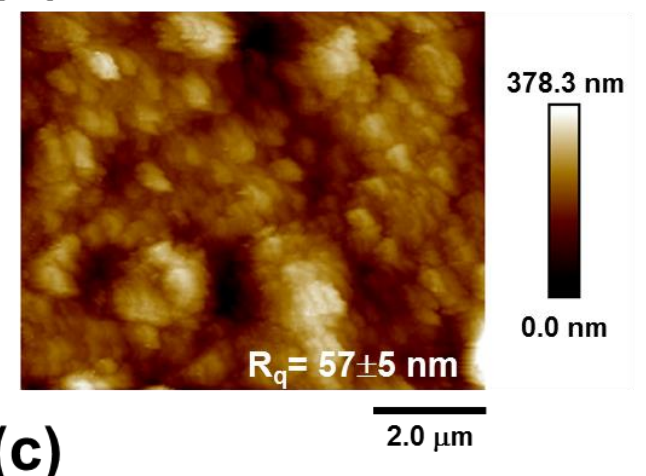

(c)
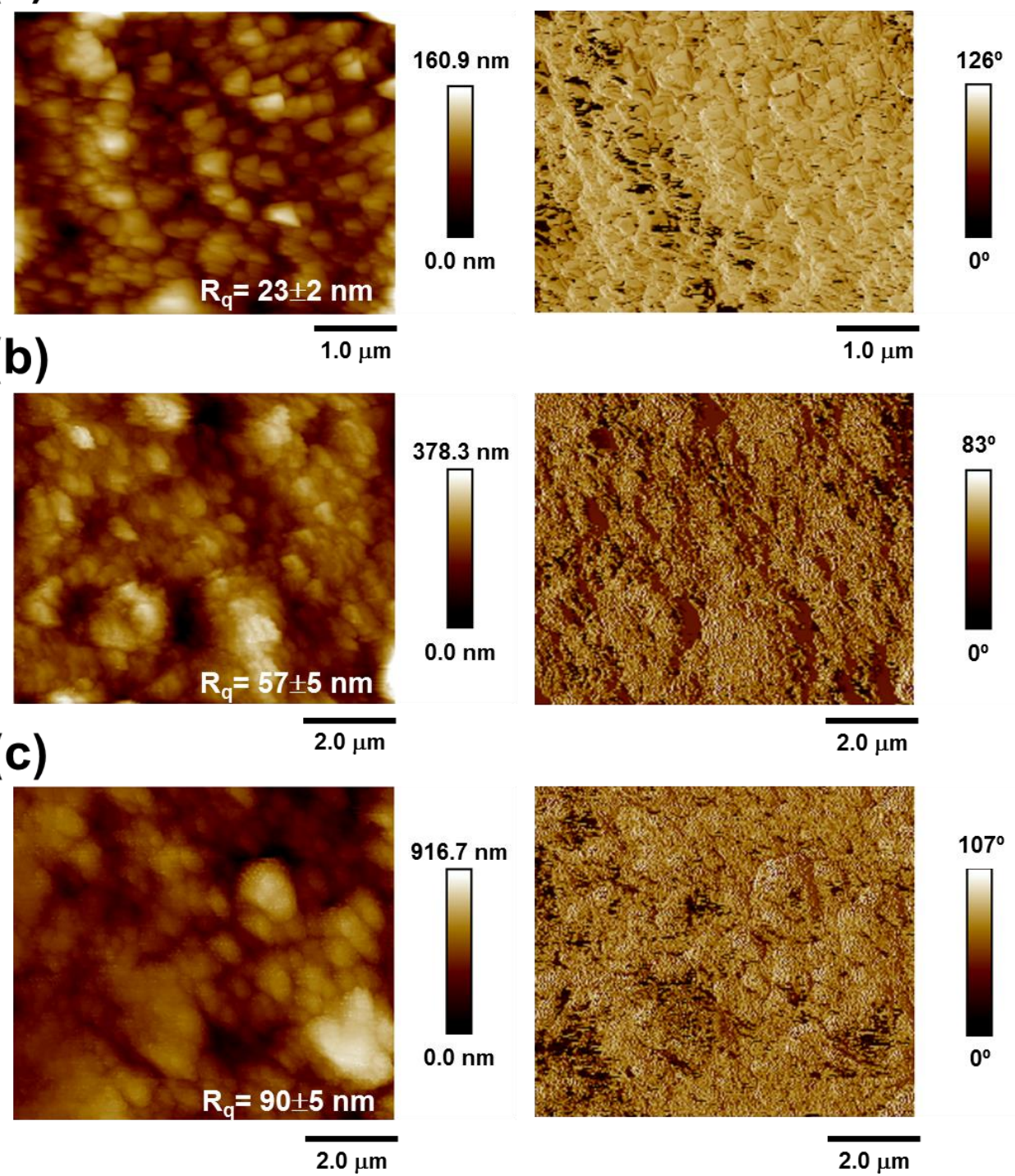

$83^{\circ}$
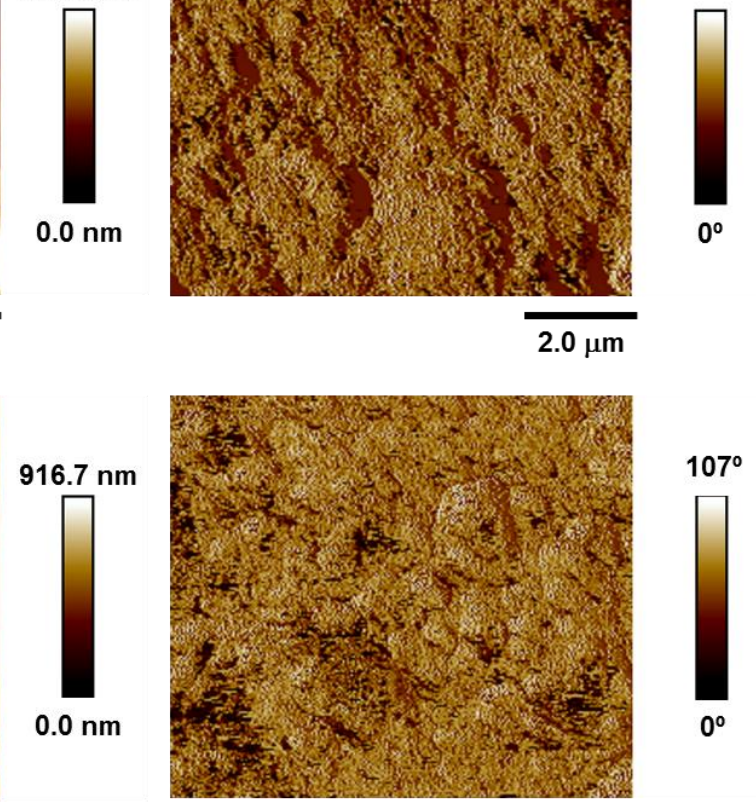

$107^{\circ}$

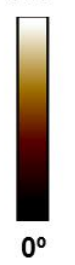

$2.0 \mu \mathrm{m}$

Figure 3 
(a)

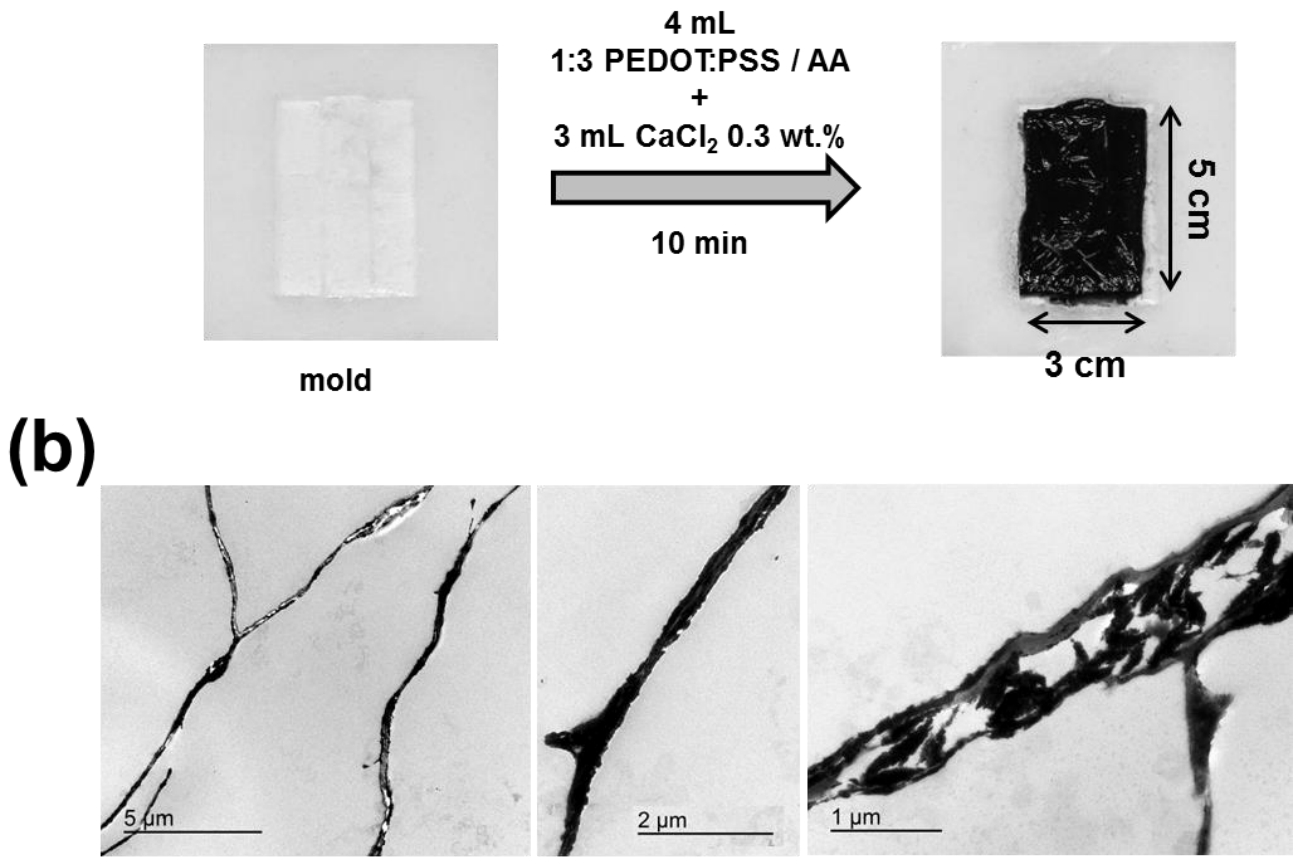

(c)

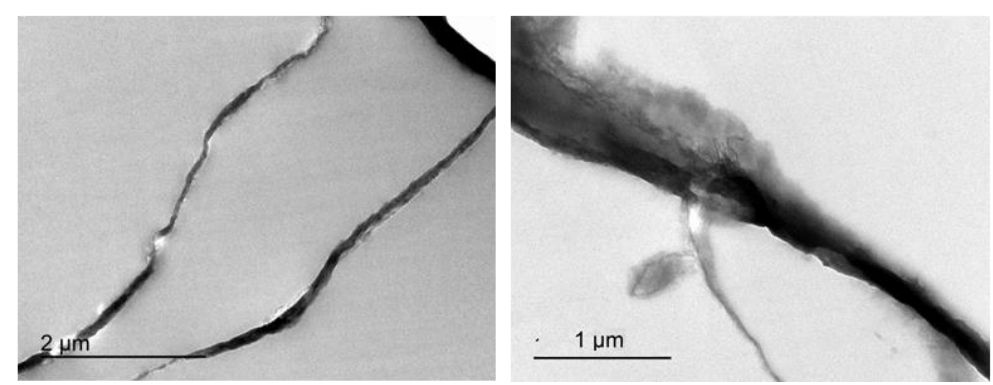

Figure 4 
(a)

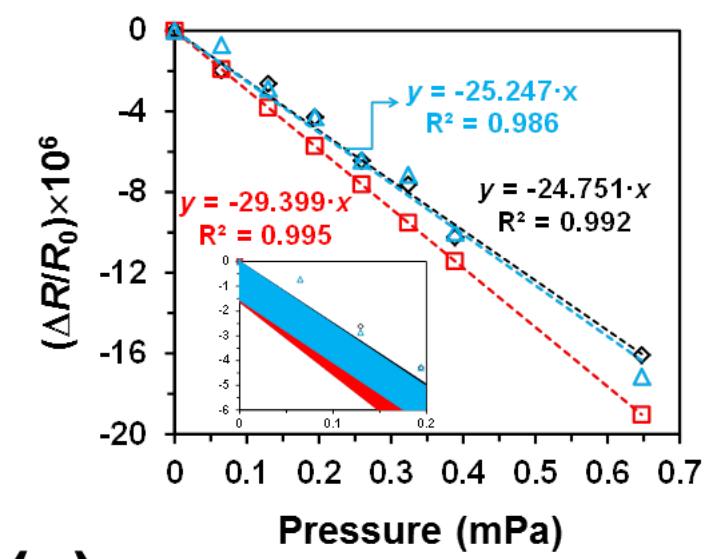

(c)

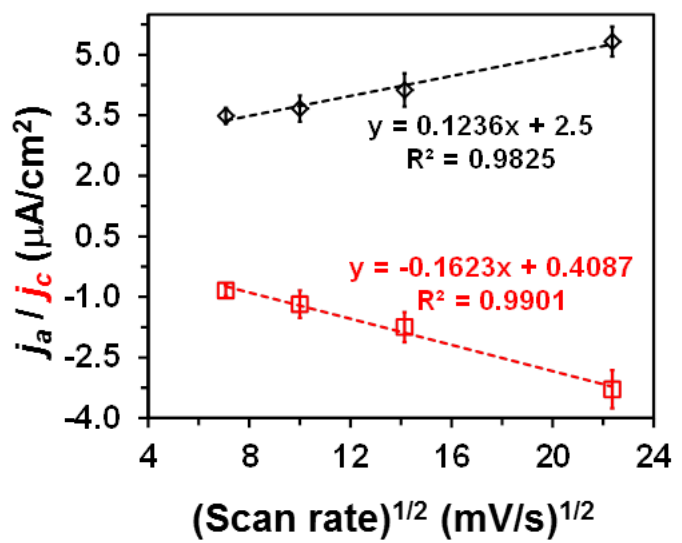

(b)

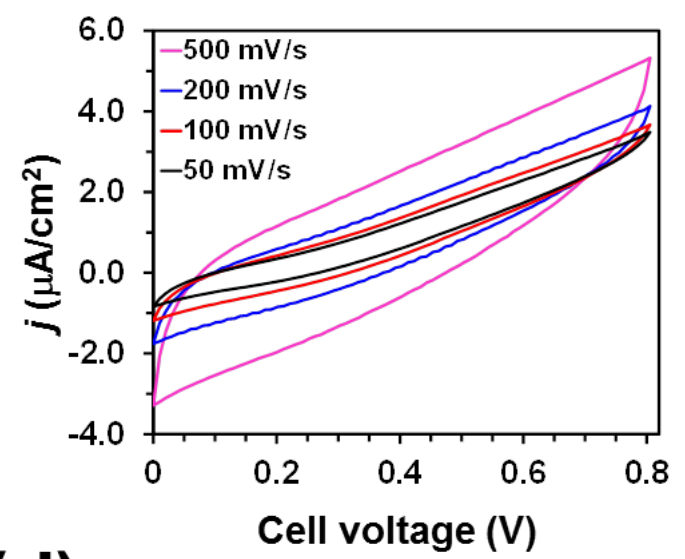

(d)

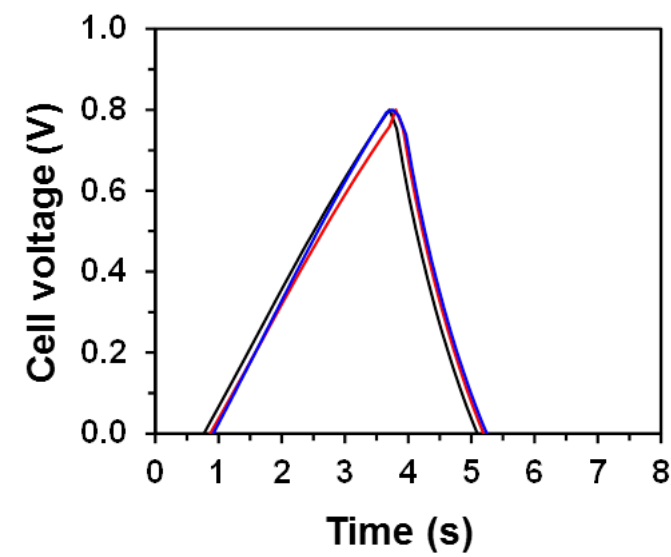

Figure 5 

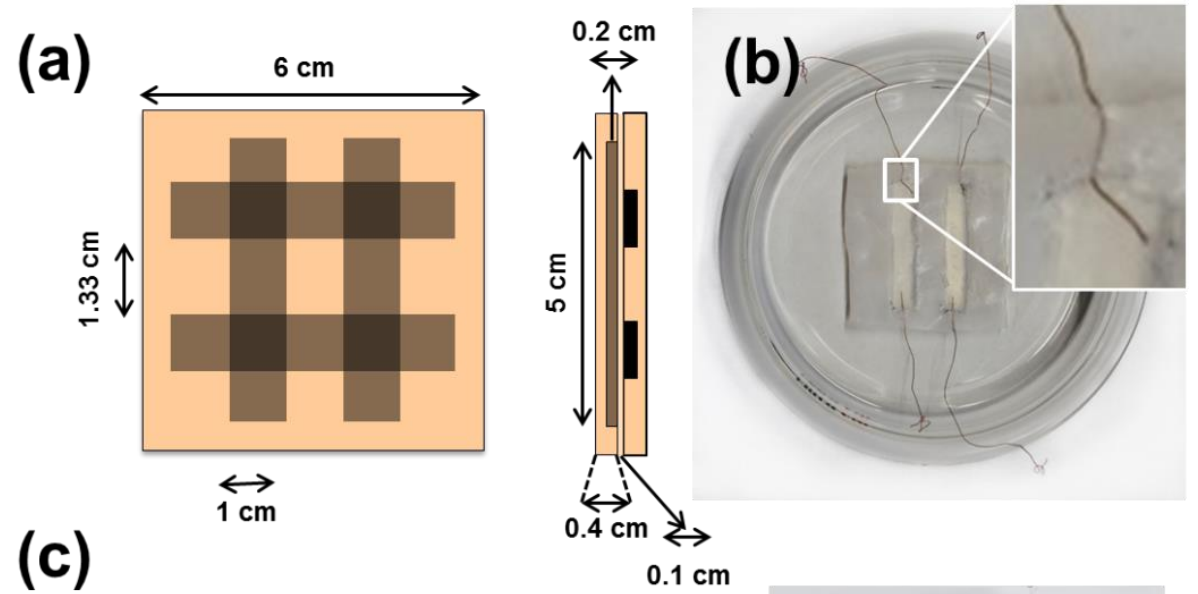

(c)

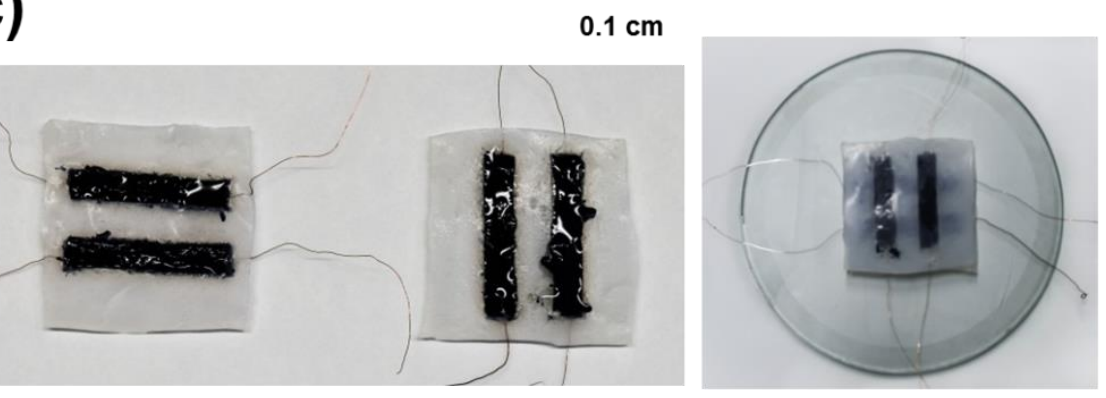

(d)
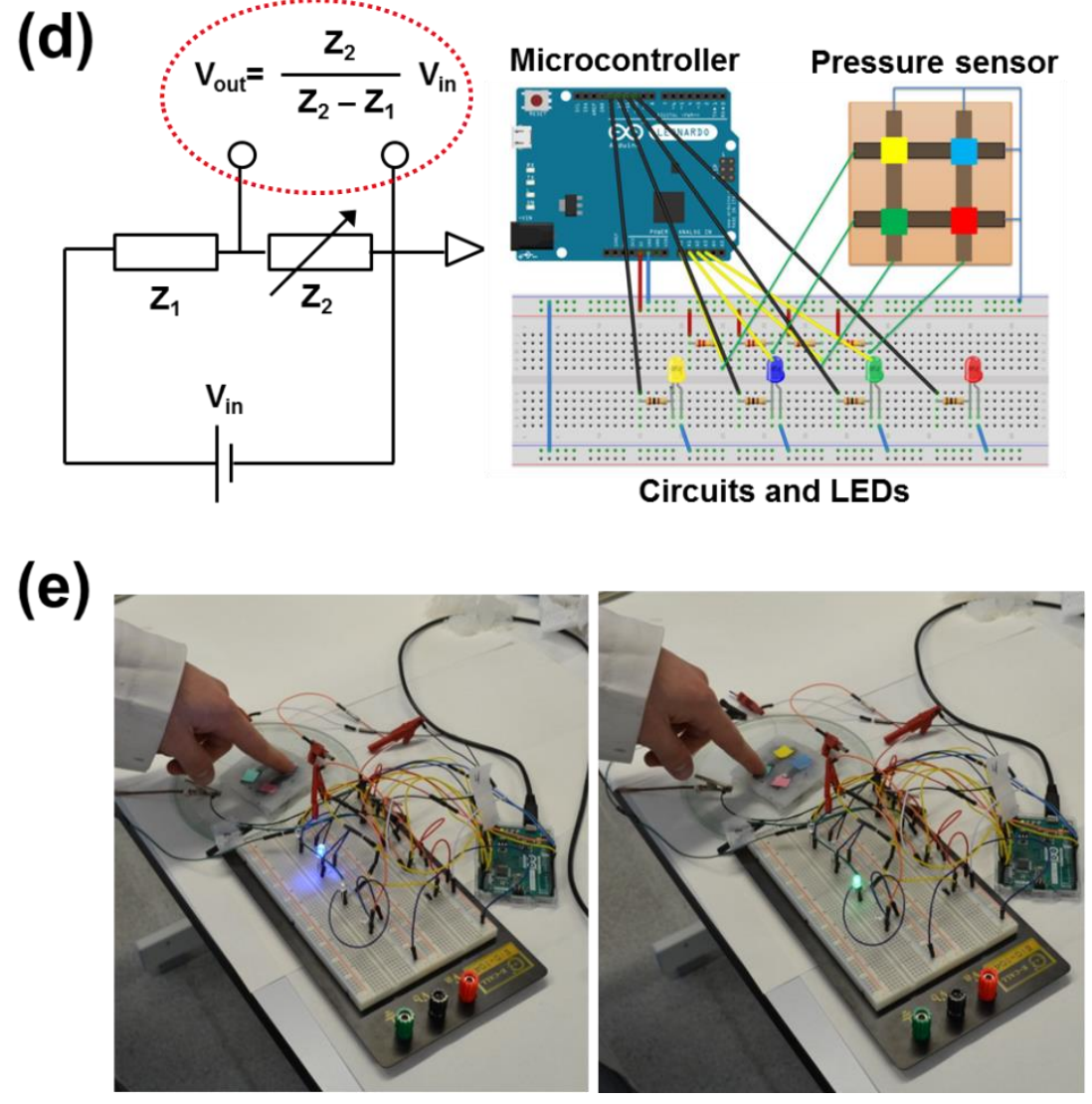

Figure 6 
(a)

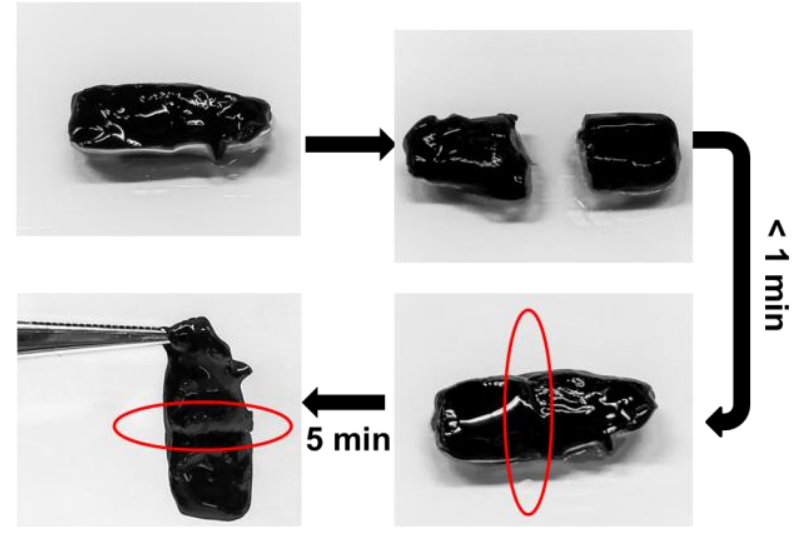

(c)

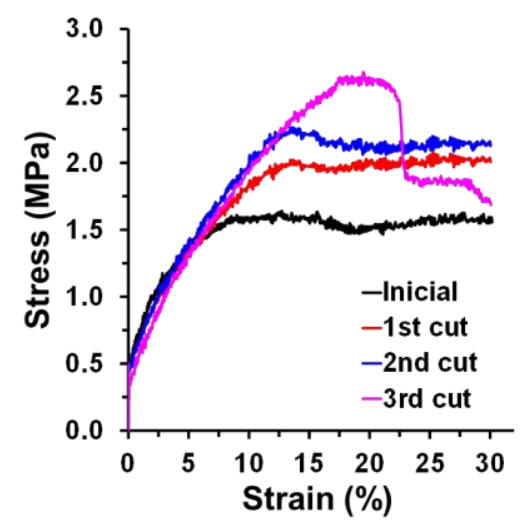

(d) (b)

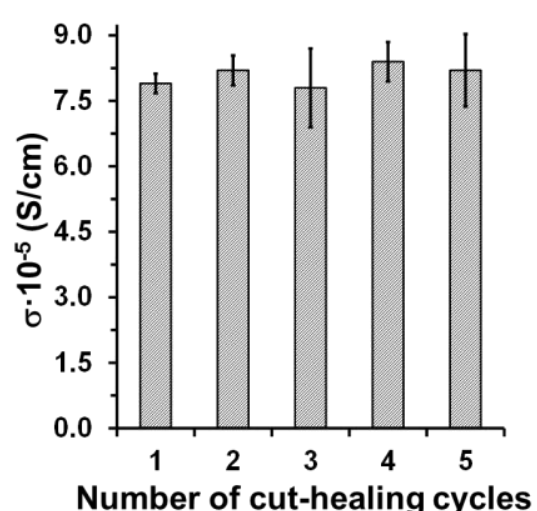

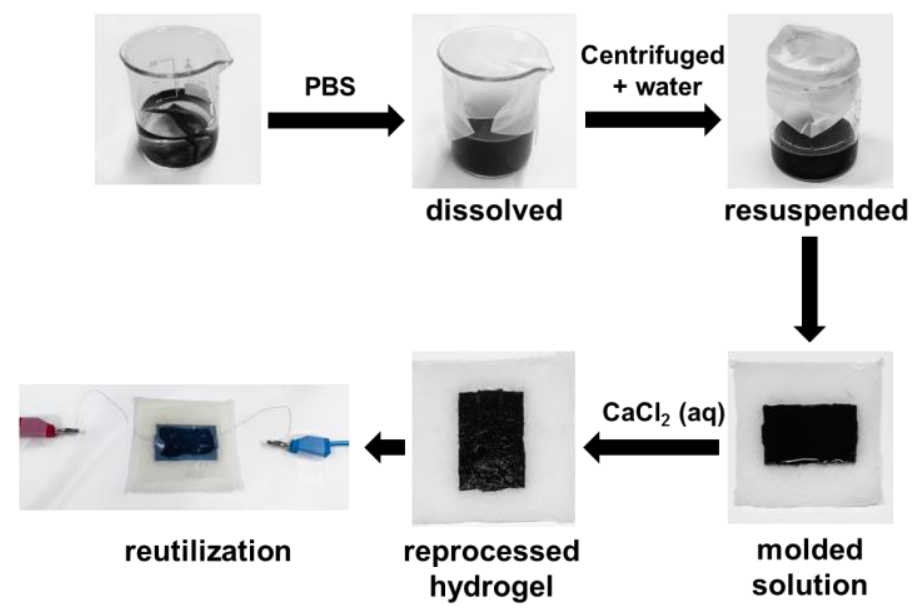

Figure 7 
Graphical abstract

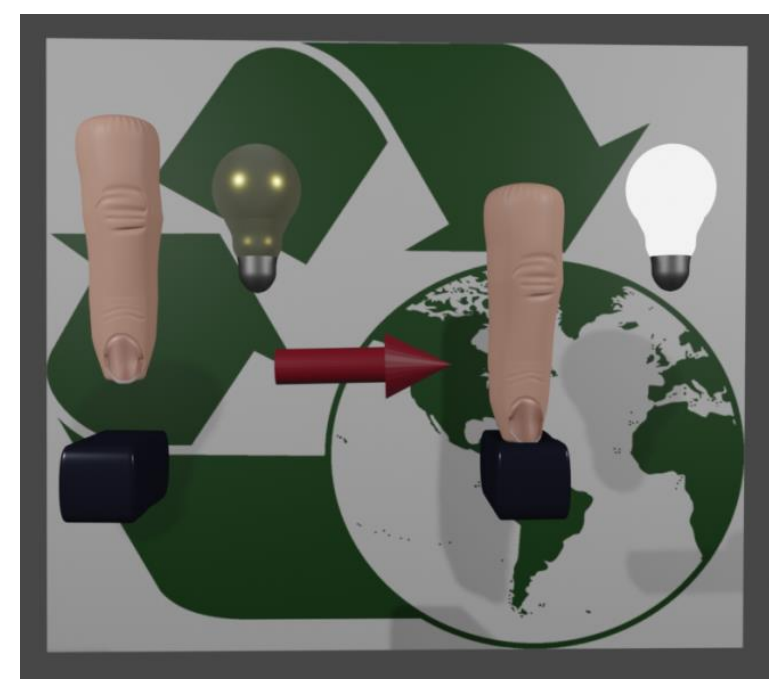

